\title{
EIS/GWM - An Integrated Automated Computer Platform for Regional Multimedia Environmental Audits
}

\author{
D. L. Toth \\ US EPA, Region III, \\ 841 Chestnut Bldg., Philadelphia, PA 19107-4431, \\ tel. (215) 597-6688, FAX (215) 597-7906
}

\begin{abstract}
In this age of global budgetary constraints, environmental audits at the regulatory level must become efficient, effective and bold. EIS/GWM-"Environmental Impact System / Geomedia Waste Contaminant Migration" is an innovative PC computer platform that integrates data bases and contaminant migration simulation models, and offers an efficient way to establish an intelligent information system to perform a variety of remediation and health exposure studies and regulatory audits.
\end{abstract}

\section{Keywords \\ Risk based environmental remediation, Regional environmental audits, automation}

\section{INTRODUCTION}

The traditional way of performing environmental permit application reviews consist of appointing a Remedial Project Manager (RPM) per site and reviewing each site on an ad hoc basis. Little attention is paid on whether each site is influenced by, or affects other sites, or what would be an environmentally sound and an economically feasible approach. Consultants often times confuse client loyalty with personal gain, and end up proposing solutions which are both environmentally disastrous and economically undesirable. Regulatory Agencies must rise above partisan rhetoric and show in deed the way of a balanced environmental stewardship, all within the constraints of dwindling budgetary resources. The way out of this impasse is through the increased efficiency that the communications revolution has brought to bear. This paper illustrates the use of one software, EIS/GWM, in support of multimedia environmental audits.

\section{Contaminant Migration and Remediation under EIS/GWM}

Remediation and containment are the most common enabling technologies for the immediate control of the spread of contamination, and for the long-term remediation strategy, especially where natural degradation is allowed to play a constructive role. 
Determination of the overall best remediation strategy is the objective of comprehensive Remedial Investigation/ Feasibility studies. These two complementary investigations represent respectively, the diagnostic and the prognostic aspects of the remediation process. The Remedial Investigation (diagnosis) is conducted concurrently with the Feasibility Study (prognosis), and emphasizes data collection and site characterization. The data collected during the diagnostic phase of the study are used to evaluate the existing state of the site, and to support the analysis and decision-making activities of the feasibility study, including the formulation of remedial alternatives. Comprehensive geomedia rehabilitation must include both aspects of the solution.

EIS/GWM is a prototype integrated environmental modeling platform, written for the MS Windows-3.x, to automate and support all aspects of the remediation problem. In this paper we summarize the sallient features of the platform, its architecture, and its operating environment. We also show pertinemt case studies where use of the platform not only streamlined the analysis phase, but also led to the most economical compliance solutions.

EIS/GWM offers a systematic, hierarchical approach, for the assessment of the relative impact of the various processes, by supporting and facilitating the analysis of the complex interactions. EIS/GWM manages the collection and use of all geographic, physical, and chemical data of a case study, entered in a graphical mode on-screen. It is used to analyze the fate and effect of chemicals in geological media. The results of this approach contribute to the better understanding of the physicochemical processes in underground flow and transport through the soil media; are useful in assisting Environmental Regulatory Agencies; and provide an integrated approach to the overall Risk Assessment of environmental contamination.

The EIS/GWM Platform offers unique capabilities to address both prognostic and diagnostic phases of remedial studies in a most efficient, reliable and cost-effective way. The basic activities of the Remedial Investigation and Feasibility Studies and their interdependency are illustrated in Figure 1. This overall methodology represents a systematic approach to achieving desired and reachable decontamination levels. The EIS/GWM Platform is the multimedia electronic integrator at the core of Figure 1, which includes:

1. Electronic data management to automate the flow of information, and streamline the overall effort and cost;

2. Health and safety planning to minimize risk of exposure; and,

3. Communications, to disseminate to the affected communities in the most efficient way the progress of the remedial studies using multimedia.

All these components are linked together in a uniform MS-Windows environment, providing task integration, the ultimate tool for every environmental auditor. These computer aided tools bring together and intelligently support various types of data used in environmental risk assessment studies, including: data collected from measuring devices in the field; information from private and public databases; scanned images; raster and vector satellite images; statistical data and risk maps.

\section{CONTEXT OF THE CONTAMINANT MIGRATION PROBLEM}


The EIS/GWM platform is based on a sound theoretical framework encompassing geostatistics, scale operators, and simulators. The formulation of the Remediation Problem is straightforward: determine the concentration ' $\mathrm{C}$ ' of a given contaminant at all spatial locations of interest over time and propose remediating measures. What makes this determination difficult is the large number of: 1/ Interacting Geologic features (aquifers, geologic faults, aquitards, rock and soil formations), and 2/ Interacting Contaminant migration processes (biochemical processes). The platform excells in handling the different natural interactions that are the key elements of the groundwater remediation problem, by managing, accurately representing, and controling the number and the degree of these interactions under the requirements of a remediation investigation.

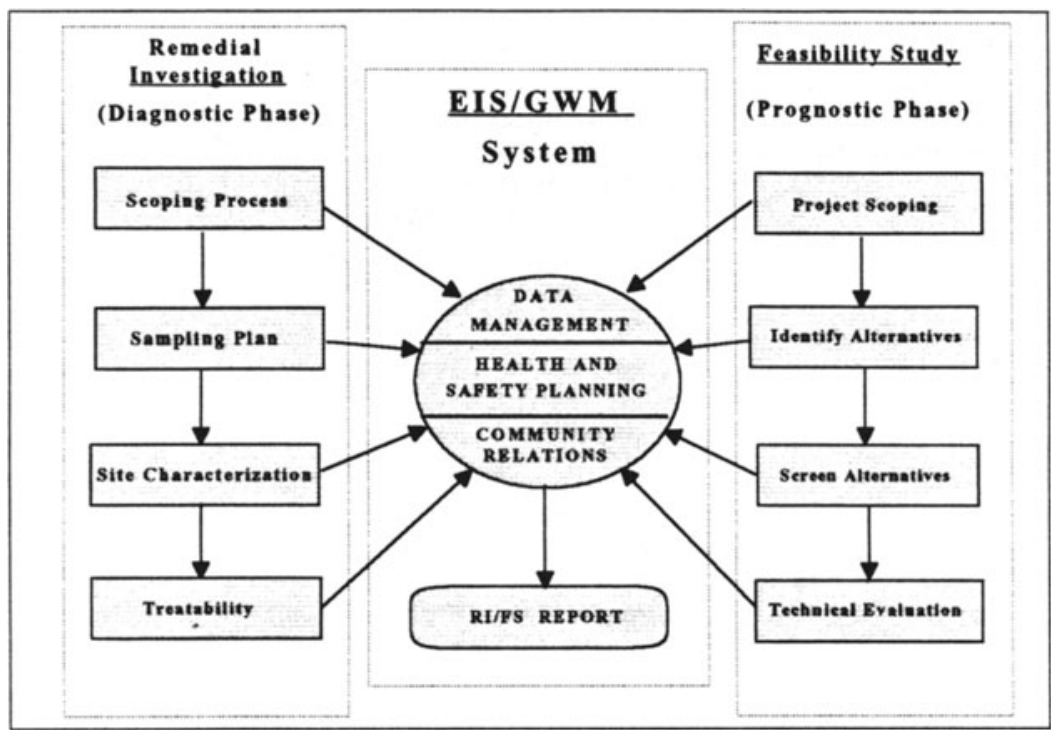

Figure 1. Interdependence of Diagnostic and Prognostic Phases of the Study.

The problem characteristics, in relation to the control volume and its impact to the overall solution, are illustrated in Figure 2. The control volume identifies the representative scale in which these interactions take place incorporated in a set of governing equations describing the balance of energy and mass over this confined domain. Different scales are necessary to accurately describe the evolution of different natural processes. What makes the EIS/GWM platform so effective is that the simulation framework is capable to automatically select the appropriate scale based on the information produced by the input (diagnostic) phase of the study. This information includes the constitutive parameters (properties) of the geomedia and the contaminants; and the influence of the ambient natural processes to the potential contaminant migration. 
Clearly, the quality of this information has an impact on the simulation (prognosis) phase, where predictions are made on the fate and migration of different contaminants. This phase is critical to the success of the overall geostatistical effort and requires selection of a 3-D grid, characterizing the topology of the macro-element discretization of the natural domain. The inference engine is the processor which distributes the properties of the media throughout the grid. Finally, the integrator is at the core of the system, operating at the level of each macro-element and taking into account the effects of all the natural processes and their interactions described at the macro-scale and micro-scale levels.

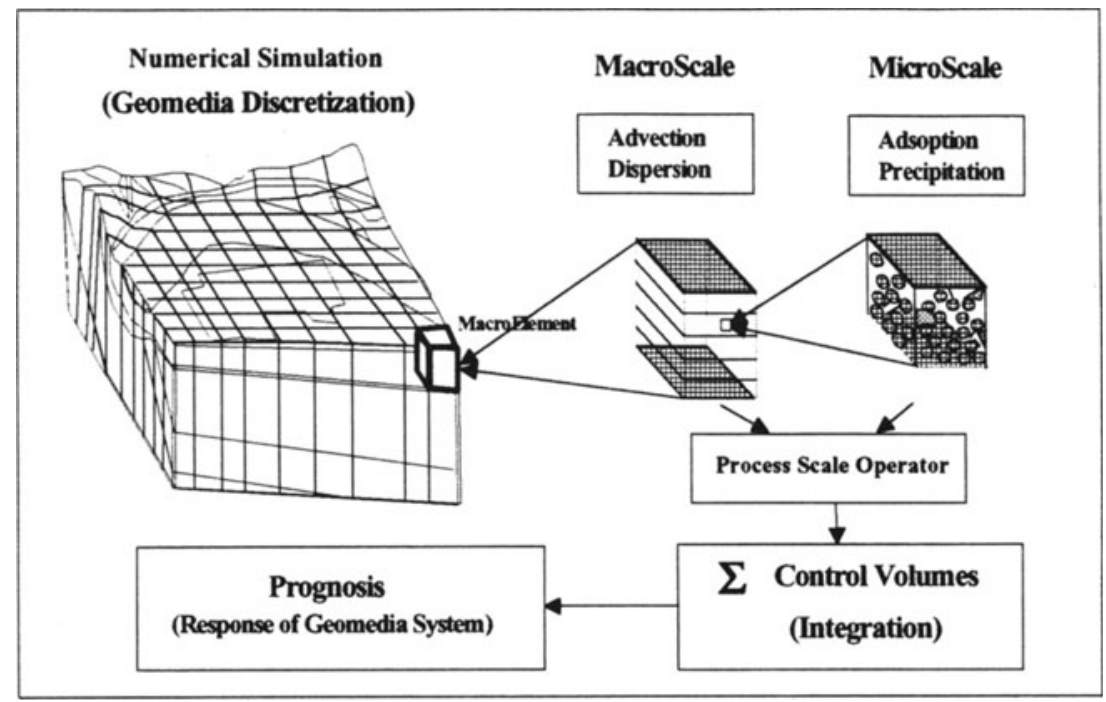

Figure 2. Soil media discretization and EIS/GWM scaling configurations.

\section{OVERVIEW OF EIS/GWM AUTOMATED PROCEDURES}

The automated procedures of the EIS/GWM platform are based on a macroengineering framework, Figure 3. An informational network identifies the pertinent modeling features associated with each grid element and integrates all process interactions occurring within each macroelement using the appropriate balance equations. These equations reflect the interaction of the different natural processes that the user selected in the input phase. The contribution of each macroelement is therefore automatically incorporated in the informational network of the EIS/GWM platform at the location of each informational node in relation to the macroelements.

This computational framework allows the generation on-the-fly of the global set of matrix equations characterizing the simulation process. With the data properly entered and kriged, all that is left to do before activating a simulation is the selection of the initial conditions. Activation of the simulation and viewing of the results are 
easily performed with a click of the mouse. If the results are not satisfactory, several options are offered; the user can either change the simulation domain, alter the loading parameters, readjust the simulation grid, or redefine the initial conditions. The beauty of the platform is that these changes and alterations are built on-the-fly, without the need to reenter any of the fundamental data. The platform cleverly assists the user in each step, and keeps track of all the new parameters that enter the simulation. At the heart of all these automated operations is a process scale operator that synchronizes all interactions first at the level of the macroelement and second, at the global level of the entire site.

\section{Process Scale Operator (Heart of the Scientific Engines)}

At the heart of the macroengineering approach is a time-and-space synchronization mechanism under the control of the Process Scale Operator. This operator controls the integration at the microscale and macroscale levels for each natural process retained for the simulation within the domain of the macroelement grid. The starting point is the determination of the microscale (control volume) in which the natural processes influence the contaminant migration in the geomedia. These natural processes govern the fate (short and long term distribution) of the chemicals in the geomedia (air, soil, water, biota).

Managing all these components for both the diagnosis and prognosis phases of a study necessitates a macroengineering framework that allows an automatic:

- classification of the natural processes and their characteristic properties affecting the contaminant migration during the diagnosis phase of the study (site investigation),

- synchronization of loading parameters,

- synchronization of time scales and integration of the natural processes at the macroelement level during prognosis (predictive) phase, and

- interaction of different chemicals and their natural processes.

All these operations start within the confines of each macroelement and encompass the following procedural steps:

1/ Define "Fluxes" of different natural processes governing the simulation.

2/ Assess "Balance" of fluxes with source/sink terms that include 'Chemical Reactions' and 'Loading Terms'.

3/ Integrate 'Balance Equations' throughout the volume of each macroelement.

Typical fluxes as implemented in the various simulation modules of the EIS/GWM platform are also shown in Figure 4, along with the general description of the 'Balance equations'. Once these parameters are identified in conjunction with the needs of a particular simulation, the Process Scale Operator automatically integrates these equations throughout the volume of each macroelement.

Several schemes are available to perform this numerical integration throughout the volume of each macroelement accounting for all possible modeling features such as vertical and horizontal wells, slurry walls, geosynthetic or clay liners, drains, rivers, lakes and ponds. A Discrete Element scheme is implemented in the platform, fitting 


\begin{tabular}{|c|c|}
\hline Modeling Features & $\begin{array}{l}\text { Geometric Configuration } \\
\text { Determine geometry } \\
\text { and location of } \\
\text { Modeling Features } \\
\text { using available toolbox } \\
\text { and corresponding } \\
\text { editing dialog boxes }\end{array}$ \\
\hline $\begin{array}{c}\text { Modeling Features } \\
\text { embedded in Macroelement Grid }\end{array}$ & $\begin{array}{l}\frac{\text { Automatic Definition }}{\text { of Modeling Features }} \\
\frac{\text { Belonging to each }}{\text { Macroelement }} \\
\text { No need to input } \\
\text { connectivities or other } \\
\text { tables to characterize } \\
\text { compatibility of } \\
\text { macroelements }\end{array}$ \\
\hline Local Integration of Natural Processes & $\begin{array}{l}\frac{\text { Automatic Evaluation }}{\text { of all interactions }} \\
\frac{\text { occuring within each }}{\text { Macroelement }} \\
\text { Integrate balance } \\
\text { equations of different } \\
\text { natural proccesses } \\
\text { interacting within each } \\
\text { macroelement }\end{array}$ \\
\hline Global Integration of Natural Processes & $\begin{array}{l}\text { Evaluate Interaction } \\
\text { of all Macroelements } \\
\text { representing the Site } \\
\text { Automatic evaluation } \\
\text { of the governing } \\
\text { equations at the global } \\
\text { scale (Matrix Form) }\end{array}$ \\
\hline
\end{tabular}

Figure 3. Built-in automated procedures under the EIS/GWM platform. 
the requirements of the macroengineering approach, with an exhaustive library of macroelements to allow an automatic integration of all pertinent features and processes interacting within the confines of each macroelement. Compatibility of pertinent physical parameters between adjacent macroelements (for example energy fluxes, and chemical concentrations), is closely examined at regular time intervals by the appropriate time marching algorithm, which is an integral part of the Scale Control Operator. This approach assures a comprehensive solution to the contaminant migration problem.

Loading parameters for a contaminant migration simulation can be either derived from the natural climate cycle (Hydrologic Cycle), or be caused by a man-made activity, pollution, accident, or a remedial action. The time variation of the intensity of these loadings is of great importance to the study. The macroengineering framework keeps track of the relative importance of these parameters in an automatic fashion. For example, the simulation of the fate of a chemical is different in the case of a dissolved trace-level contaminant from the case of a large scale spill release.

Both, the release (source) mechanism and the evolution processes of the contaminant (advection, dispersion, reactions) are affected by the time incrementation used in the simulation; and the model automatically identifies the proper time discretization satisfying all requisite criteria (e.g. Courant conditions).

But perhaps the most important aspect of the platform automation is the ability it provides the user to experiment with the simulation of the groundwater contaminant migration. The EIS/GWM platform frees the hands of the user to make changes on his model without being penalized. Modifying for example the grid density of the macroelement to better simulate the contaminant source migration is as simple as making a spelling correction with a text editor. EIS/GWM offers the user the power to alter the grid using a graphical interactive mode that automatically adjusts on the spot the connectivities of the simulation module as follows. To change the grid you use option "Edit Grid" from menu "Grid", and select with the mouse from the toolbox the vertical arrow icon. Then, you move to the desired location in the working window, where you wish to add a column of macroelements, you click on the mouse: it is as simple as that. The example case study below showcases the platform capabilities.

\section{ADVANCED BIODEGRADATION CASE STUDY}

The site under investigation covers an area approximately $3,000 \times 3,000 \mathrm{ft}$. and is seated on a plateau-like bench formed by river deposits of an ancient river. It is entirely developed, including paved areas, residential housing, and some grassy areas. A fairly well developed storm sewer system is believed to collect most of the local runoff from rainfall. An LNAPL (light non-aqueous phase liquid) fuel has been released into the soil and the shallow groundwater at the site near two oil tank farms. A detailed bioremediation study is initiated to predict the extent and migration of the spill shown in Figure 4. Different remediation scenarios are also examined.

There are two deeper aquifers at the site, but little information is available about them. However, the contamination is believed to be limited to the shallow 
(unconfined) aquifer, which is the subject of this investigation. The only available information on depth and thickness of the geologic formations is given for cross-sections $\mathrm{A}-\mathrm{A}^{\prime}$ and $\mathrm{B}-\mathrm{B}^{\prime}$, respectively in the general direction of flow and normal to it. In these cross-sections, the shallow aquifer is portrayed as limited to the sandy stratum which is underlain by clayey and sandy silt deposits. These deposits likely form an aquitard stratum separating the shallow aquifer from the Sunset aquifer. Samples collected from deeper pockets of clayey sand indicate that contamination may have reached these depths. Because the site is fairly well developed with paved areas and with a storm sewer system, it is believed that most of the recharge takes place upgradient of the site.

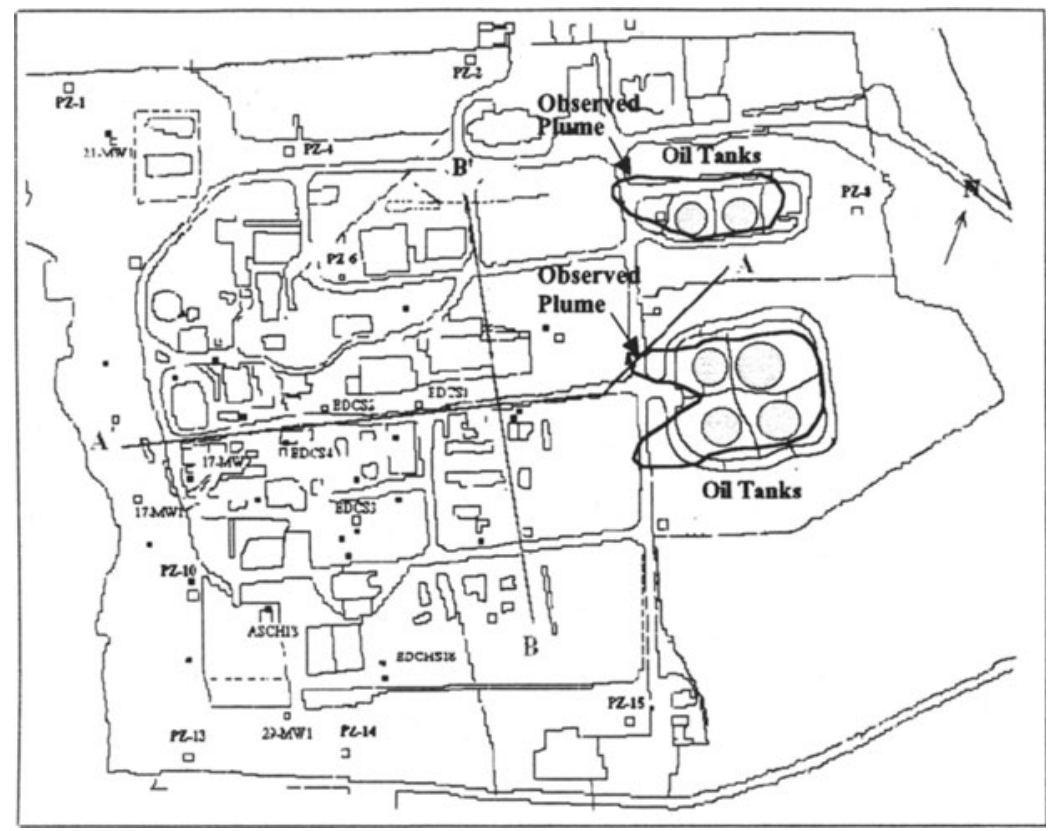

Figure 4. Site layout and cross section location.

The groundwater flow is generally in SouthWest direction. However, the "V" shaped delineation of the south contaminant plume necessitates a closer examination of the local flow regime west of the oil tanks. A previously "calibrated" flow model does not explain the bifurcation of the plume. In the present study we conduct a more refined investigation to verify the existence of a preferential flow path. In this instance the observed plume will be used as a tracer to explore the groundwater flow regime, assuming that advective conditions prevail at the site.

Ground water flow in the shallow zone is in the SW direction, with an average gradient of $5 \mathrm{ft}$ per 100 horizontal feet. A local site investigation west of the main group of oil tanks revealed the existance of burried inactive conduits that could 
impose a preferential flow path, strong enough to alter the shape of the contaminant plume. The porosity of the aquifer has not been modified. The selected value of 0.25 is at the low end of values listed in the literature and seems adequate. The low value favors faster seepage velocities, therefore it is on the conservative side of the analysis. This argument alone is not sufficient to justify the selection, because ultimately one must show that the model used is capable of reproducing both observed piezometric heads and contaminant plumes.

This is where the different modeling tools of the EIS platform become handy. To model the preferential flow path created by the buried conduits a $20 \mathrm{ft}$. wide vertical trench (flow discontinuity) is selected from the toolbox and placed at the location of the old conduits as shown below. Then the properties of this trench are specified. A high hydraulic conductivity is selected to reflect the preferential flow regime. And that is all we need. The new flow simulation does not change the overall pattern of the piezometric heads. Only the velocity field is affectede in the vicinity of the conduits.

\subsection{Design of Bioremediation Alternatives}

The model calibration is based on the observed contaminant migration, spanning a period of one year. It is clear that an immediate containment action must be taken to stop the migration of BTEX in the downgradient direction, and protect key facilities.

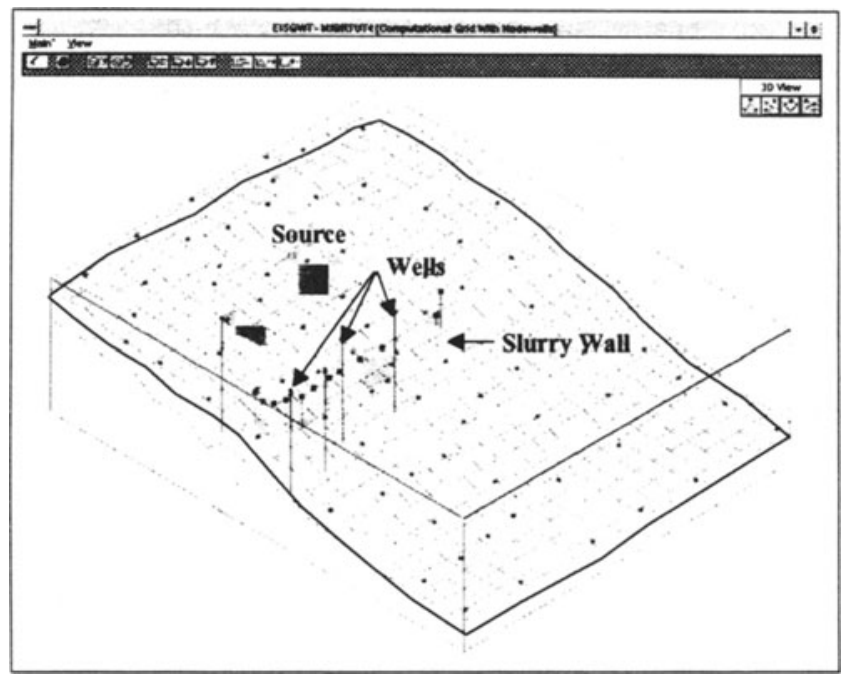

Figure 5. Slurry wall configuration.

One design alternative for containment is to build a slurry wall along the perimeter of these facilities. Then to further reduce the level of the BTEX concentrations, four injection wells are placed in front of the wall to reduce the contaminant concentrations. Oxygen with water are to be injected periodically in the wells to 
enhance natural biodegradation. The objective of the simulation is to check the feasibility of such design. The slurry wall is introduced in the model graphically through the use of slurry wall element in the toolbox. A similar approach is adopted to incorporate the injection wells, illustrated in Figure 5.

We implement this case following the steps introduced during the calibration of the model, starting with the flow module and continuing with the bioremediation simulation for 1000 days. The results of the simulation are conclusive. The shallow slurry wall is capable to contain the BTEX migration. After 800 days there are still some contaminant residuals behind the wall, but for the most part the level of the concentration has been reduced. Improvements can be made in optimizing the effectiveness of the pumps. What should be remembered is that a bioremediation model should be based on a rational well thought-out plan that includes:

- a clear definition of the goals of the study,

- an assessment of the quality of the site specific data, and an understanding of the limitations and quality of the results.

\section{CONCLUDING REMARKS}

In this article about the EIS/GWM platform and its use for environmental audits we have made the following points:

- Simulation models are necessary for ground water pollution remediation.

- Pumping tests provide local (intermediate scale) estimates of aquifer hydraulic properties and should not be confused with models.

- For proper use in predicting future system behavior, the model must be calibrated and verified. Calibration is the tuning of the model's parameters to match one set of observed conditions. Validation is the proof that the model predicts another set of conditions without need of further tuning.

- A good ground water biodegradation model is a suite of integrated simulation modules (flow between aquifer cells, recharge, pumping, ET, connection with surface waters, dispersion, chemical reactions, biodegradation) which render the calibration exercise rather arcane: an art form requiring substantial experience, reserved for the few.

- The EIS/GWM platform has made this art form accessible to everyone.

- This is the only platform suporting multiple plume 3-Dimensional simulations.

In this approach, not only does the regulator find distilled a multi-year experience in modeling and calibration, but he also finds the tools to use this experience tangibly in the form of the EIS/GWM platform.

\section{REFERENCES}

1. MicroEngineering, 1994. "BIOREM-3D, Contaminant Biodegradation Models Under the EIS/GWM Platform," Report No. Mi-94-M009.

2. MicroEngineering, 1994. The EIS/GWM Intgrated Computer Platform: "Environmental Impact System for Contaminant Migration Simulataions and Risk Assessment," Report No. Mi-94-M001. 